\title{
Dynamics of Big Bubble Formation During Deep Anterior Lamellar Keratoplasty in Eyes with Advanced Keratoconus
}

This article was published in the following Dove Press journal:

Clinical Ophthalmology

Mohamed Bahgat Goweida (1D)

Amr Abuelkheir (D) ${ }^{1,2}$

Wael Abdel Rahman El-

Menawy ${ }^{1,2}$

Shahira Mahmoud (iD) 1,2

'Department of Ophthalmology, Alexandria Main, University Hospital, Alexandria, Egypt; ${ }^{2}$ Faculty of Medicine, Alexandria University, Alexandria, Egypt
Correspondence: Shahira Mahmoud 4th Ghanem Abd El- Reheem Street, Fleming, Alexandria, Egypt Tel +20 I229557 I3 I

Email Shahira.rashad@alexmed.edu.eg
Purpose: To study the dynamics of big bubble (BB) formation in eyes with advanced keratoconus (KC) during deep anterior lamellar keratoplasty (DALK).

Methods: A retrospective review of medical records and videos of DALK surgeries performed during the period from January 2013 to December 2019 on keratoconic eyes. Eyes with formed BB, in the presence of a relatively clear stroma, were included. We studied the following parameters the location of BB commencement, duration from the start of the bubble until complete formation, and the pattern of air passage during the formation process. Results: A total of 37 eyes met the inclusion criteria. Type $1 \mathrm{BB}$ was formed in 22 eyes with a mean formation time of $1.9 \pm 1.1$ seconds and a mean bubble diameter of $7.6 \pm 1.1 \mathrm{~mm}$. Type 1 BB started in the center as a small dome expanding centrifugally with preceding localized stromal whitening in 19 eyes and without whitening in three eyes. Type 2 BB was formed in 14 eyes with a mean formation time of $0.3 \pm 0.12$ seconds and a mean bubble diameter of $7.4 \pm 1.8 \mathrm{~mm}$. Type $2 \mathrm{BB}$ started in the center in 13 eyes and the mid periphery in one eye, through either direct access to the plane between Descemet's membrane and the pre-Descemet's layer in six eyes, or preceded by a small intrastromal bubble in eight eyes. Mixed bubble was observed in one eye, which started as a small central Type 1 BB followed by the rapid formation of a Type 2 BB.

Conclusion: Eyes with $\mathrm{KC}$ are associated with central commencements of both Type 1 and Type 2 BB during DALK. Duration of BB formation was significantly faster in Type 2 BB, with most starting as a small intrastromal bubble before air gain access to the plane between DM and stroma.

Keywords: DALK, type 2 big bubble, Descemet's membrane

\section{Introduction}

Deep anterior lamellar keratoplasty (DALK) has replaced penetrating keratoplasty in treating corneal stromal pathologies with healthy endothelium such as keratoconus, scars, stromal dystrophies and degenerations. ${ }^{1-3}$ The big bubble (BB) technique is considered the most popular technique used in DALK, in which the air is injected in the corneal stroma to achieve separation at the posterior lamellae. ${ }^{4-6}$

The plane of cleavage during $\mathrm{BB}$ formation has been a topic of interest in many publications. It has been believed previously that BB DALK is a Descemet's membrane (DM) baring technique. However, many studies have recently confirmed the presence of a stromal sheet (pre-Descemet's layer [PDL]) covering DM after BB formation. ${ }^{7-9}$ Consequently, three types of BB have been described. Type 1 BB (white margin), which 
is the most common, forms between the stroma and the PDL. Type 2 BB (clear margin) forms between DM and the PDL and has previously been believed to form intra-DM between the banded and nonbanded zones. ${ }^{10}$ The third type is the mixed BB, in which both Type 1 and Type 2 appear together, either complete or partial. ${ }^{9}$

The dynamics of BB formation have been studied in vitro by air injection in sclerocorneal buttons. Type 1 $\mathrm{BB}$ has been described as starting as tiny central bubbles anterior to DM, which coalesce to form a dome-shaped BB expanding centrifugally to a mean diameter of $8.11 \mathrm{~mm}$. On the other hand, type 2 BB starts as a thin-walled bubble in the periphery and expands as a thin transparent dome with a mean diameter of $10.2 \mathrm{~mm}$. Mixed type bubble occurs during the formation of Type $1 \mathrm{BB}$, when air leaks through the stretched fibers of the PDL at the periphery of the bubble and gains access to the plane between DM and the PDL. ${ }^{11}$

In this study the commencement locations of Type 1 and Type $2 \mathrm{BB}$, the pattern of the air passage in the corneal stroma, and the total time required for $\mathrm{BB}$ formation in eyes with advanced keratoconus (KC) during DALK using the BB technique.

\section{Materials and Methods}

We conducted a retrospective review of medical records and videos of DALK surgeries performed on keratoconic eyes during the period from January 2013 to December 2019. This study was conducted in accordance with the tenets of the Declaration of Helsinki and was approved by the Ethics Committee of the Faculty of Medicine, Alexandria University. All patients provided written informed consent.

All procedures were performed by a single surgeon, Goweida MB, using the BB technique previously described by Anwar and Teichmann. ${ }^{4}$ Some modifications of the original technique have been made, such as performing paracentesis before bubble formation for injecting small air bubbles in the anterior chamber and using a Fogla 27-gauge air-injection cannula (Bausch \& Lomb, Rochester, NY) introduced in the central or paracentral area to create the BB.

All videos were reviewed by slow motion video editing using Final Cut software on a MacBook Pro computer (Apple Inc. USA) to study the dynamics of BB formation from the start of air injection until the complete BB formation. The inclusion criteria included eyes with Type 1 and Type 2 bubbles with relatively clear stroma during all stages of BB formation, permitting clear study of the process. Videos meeting the inclusion criteria were assessed regarding the location of $\mathrm{BB}$ commencement, duration from the start of bubble until the formation, and the pattern of air passage during the process of formation. The start of the BB was described as being central (within the central $4 \mathrm{~mm}$ of the cornea), mid-peripheral (within 4-6 $\mathrm{mm}$ from the corneal center), or peripheral (if beyond the central $6 \mathrm{~mm}$ ). The size of the BB was measured relative to the size of the trephination. The duration was recorded in seconds.

The exclusion criteria included eyes with failed BB, eyes with marked stromal whitening obscuring the start of the $\mathrm{BB}$, eyes with preexisting DM tears or stromal scarring, and eyes with burst BB.

\section{Results}

A total of 286 videos of BB DALK for advanced KC were reviewed: 142 eyes had a Type 1 BB (49.7\%), 45 eyes had a Type 2 BB (15.7\%), six eyes had a mixed BB (2.1\%), and 93 eyes had a failed BB $(32.5 \%)$. Of the reviewed cases, 37 eyes met the inclusion criteria. Type $1 \mathrm{BB}$ was formed in 22 eyes, Type $2 \mathrm{BB}$ in 14 eyes and mixed bubble was formed in one eye.

\section{Type I BB}

The mean age of the patients with Type 1 BB was $25 \pm 5.6$ years. The mean preoperative central corneal thickness (CCT) and average keratometric (K) readings were 366.1 $\pm 73.9 \mu \mathrm{m}$ and $64.6 \pm 5.3$ diopters, respectively. The average trephination diameter was $8.1 \pm 0.21 \mathrm{~mm}$. A mean formation time of $1.9 \pm 1.1$ seconds was recorded from the start of the BB until its complete formation, and the mean bubble diameter was $7.6 \pm 1.1 \mathrm{~mm}$.

Two patterns were observed during BB formation. In the first pattern, 19 eyes showed stromal whitening (Video S1) starting from the location of air injection and extending to one side of the limbus (Figure 1A), followed by the appearance of the $\mathrm{BB}$ as a small dome in the center (Figure 1B), and expanding centrifugally (Figure 1C). In the second pattern, formation of the BB without stromal whitening (Video S2) was observed in three eyes starting from the center and expanding centrifugally (Figure 2).

\section{Type 2 BB}

The mean age of patients with Type 2 BB was $31.8 \pm 10.1$ years. The mean preoperative $\mathrm{CCT}$ and average $\mathrm{K}$ readings were $298.5 \pm 118.3 \mu \mathrm{m}$ and $69.6 \pm 6.1$ diopters, 


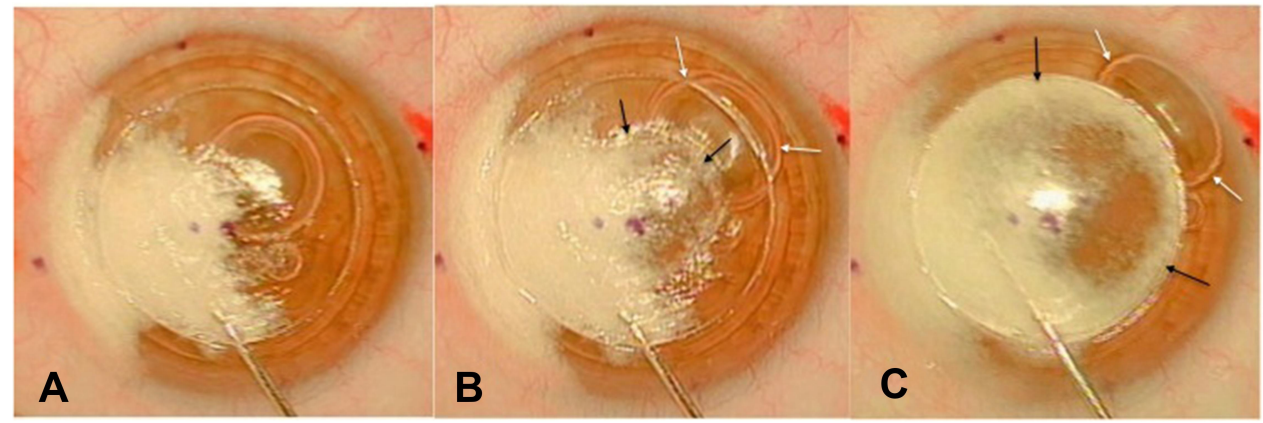

Figure I Type I big bubble formation with stromal whitening. (A) Stromal whitening extending from the site of air injection to the limbus on one side. (B) Central commencement of Type I bubble (black arrows) with displacement of the anterior chamber (AC) bubbles to the periphery (white arrows). (C) Complete formation of Type I bubble.

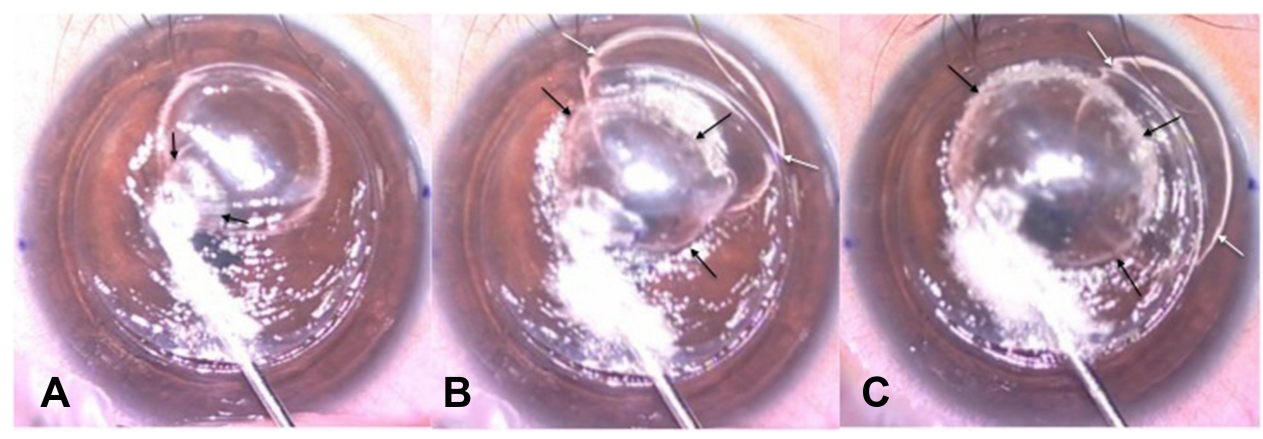

Figure 2 Incomplete Type I big bubble formation without stromal whitening. (A) Central commencement of Type I bubble in a clear cornea (black arrows). (B and C) Enlargement of Type I bubble (black arrows) with AC bubble displacement to the periphery (white arrows).

respectively. The average trephination diameter was $8.2 \pm$ $0.23 \mathrm{~mm}$. The mean time of bubble formation was $0.3 \pm$ 0.12 seconds, and the mean bubble diameter was $7.4 \pm$ $1.8 \mathrm{~mm}$. The bubble expanded from the starting point to the periphery in nine eyes, whereas five eyes showed concentric enlargement similar to that of type $1 \mathrm{BB}$. Two patterns were observed during bubble formation. In the first pattern, in eight eyes, the bubble started as a minute intrastromal bubble (Figure 3A) with sudden and rapid formation of a clear margin bubble (Figure $3 \mathrm{~B}$ and $\mathrm{C}$, and Video S3). Mid-peripheral commencement of the bubble was observed only in one eye (Figure 4 and Video S4), while the remaining seven had a central commencement. The second pattern was direct access of air to the plane between DM and PDL at the site of air injection in six eyes with a central commencement in all of them (Figure 5 and Video S5).

\section{Mixed Bubble}

One eye with mixed bubble met the inclusion criteria, in which air injection resulted in the formation of a small central Type $1 \mathrm{BB}$ with a diameter of $3 \mathrm{~mm}$, followed by the rapid formation of a Type $2 \mathrm{BB}$, starting at one edge of the formed Type 1 with a diameter of $6 \mathrm{~mm}$ (Figure 6 and Video S6). The age of this patient was 29 years. The preoperative CCT and average $\mathrm{K}$ readings were $338 \mu \mathrm{m}$ and 67 diopters, respectively. The trephination diameter was $8.25 \mathrm{~mm}$.

\section{Discussion}

Since the popularization of the BB technique in lamellar corneal surgery, the mechanism of BB formation during DALK has been an interesting research topic. The presence of stromal emphysema during the process of BB formation has resulted in some delay of the understanding of the types and mechanism of BB formation. To overcome this difficulty, the dynamics of $\mathrm{BB}$ formation have been studied on sclerocorneal buttons by stromal air injection endothelial side up..$^{9,11,12}$

However, the postulated mechanisms may not be similar to BB formation during DALK surgery owing to several reasons. First, the examined sclerocorneal buttons are considered having normal stroma, whereas surgeries are performed in pathological corneas with the expected change of the stromal microarchitecture, resulting in 


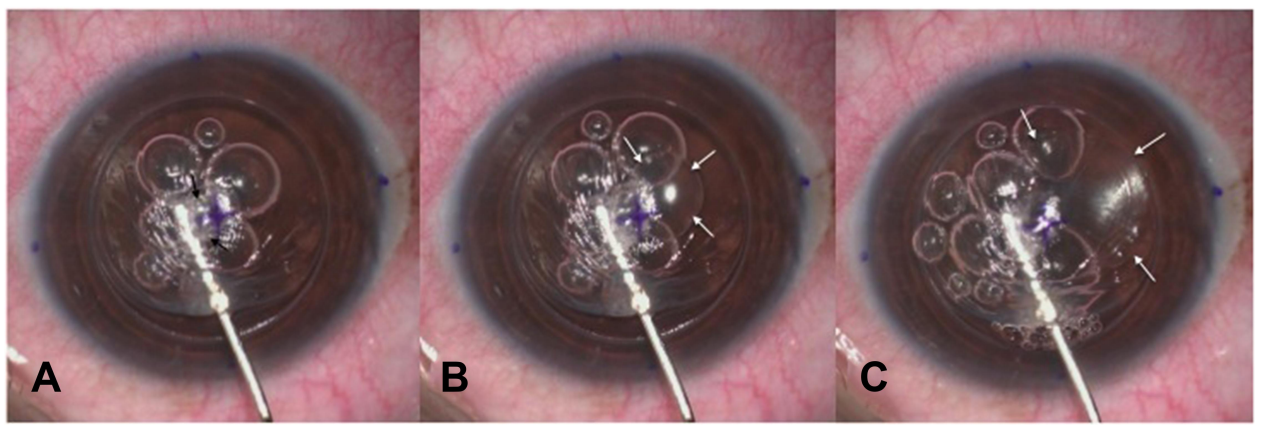

Figure 3 Type 2 bubble formation after a small intrastromal bubble. (A) Air injection with the formation of a small intrastromal bubble (black arrows). (B) Rapid formation of Type 2 bubble as a small central bubble (white arrows). (C) Centrifugal enlargement of Type 2 bubble (white arrows).

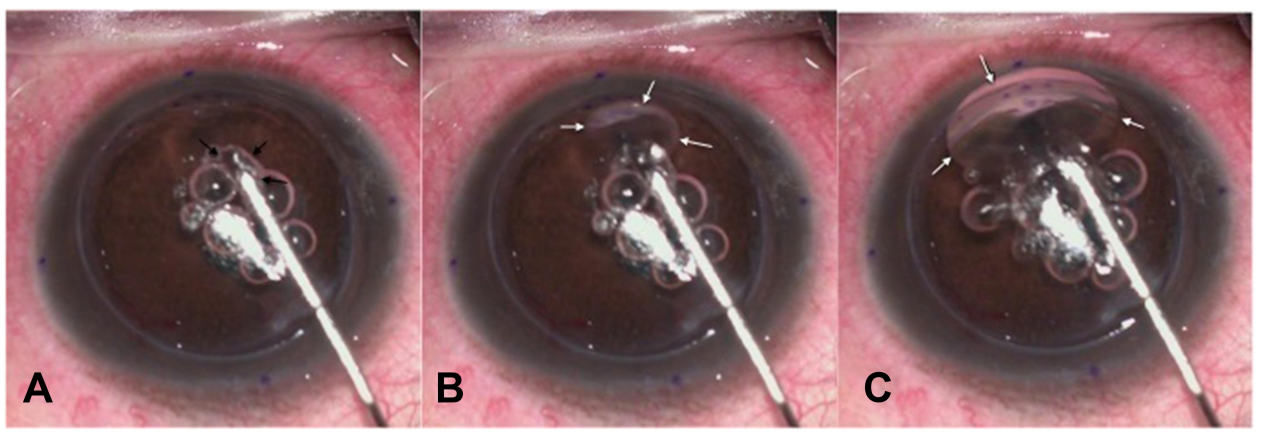

Figure 4 Midperipheral formation of Type 2 bubble after a small intrastromal bubble. (A) Air injection with the formation of a small intrastromal bubble (black arrows). (B and $\mathbf{C})$ Formation of Type 2 bubble enlarging inferiorly (white arrows).

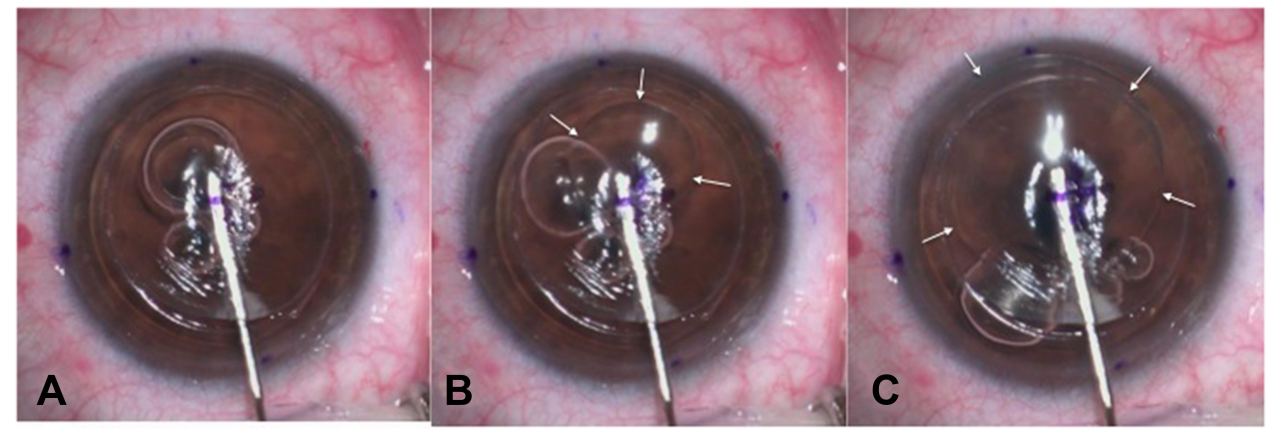

Figure 5 Direct access of air to the plane between the DM and stroma to form Type 2 bubble. (A and $\mathbf{B}$ ) Central commencement of Type 2 bubble (white arrows). (C) Centrifugal enlargement of Type 2 bubble (white arrows).

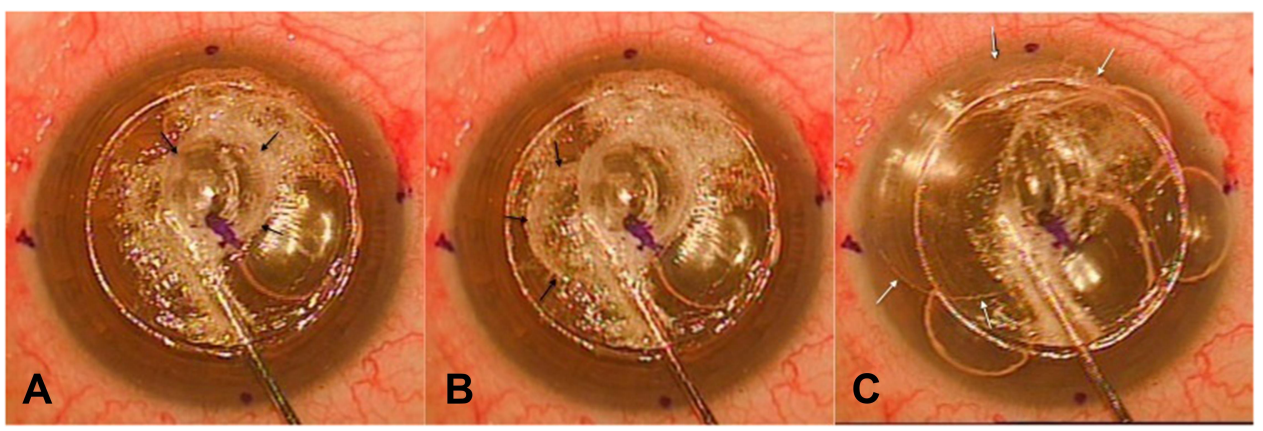

Figure 6 Mixed bubble without stromal whitening. (A) Central commencement of Type I bubble (black arrows). (B) Enlargement of the bubble towards one side (black arrows). (C) Rapid formation of type 2 bubble (white arrows). 
different mechanism of BB formation. Second, the used buttons are usually of old age donors with anticipated change in the stromal collagen, and there is a possibility of higher incidence of the formation of Type $2 \mathrm{BB}^{13}$ Finally, the placement of the buttons endothelial side-up will eliminate the counterbalancing effect of intraocular pressure during air injection.

The integrity of the PDL is considered to be the contributing factor responsible for the type of bubble formed during pneumodissection. The criteria of the PDL have been described by Dua and his associates as being 15 to 20 microns thick, devoid of keratocytes, and impervious to air. ${ }^{9}$ Normally, injected air will diffuse through the corneal stroma and on reaching the posterior lamellae, it separates the PDL from the deep stroma forming a Type $1 \mathrm{BB}$. A defect in the PDL may allow air passage to separate the DM resulting in the formation of Type $2 \mathrm{BB}$.

Previous studies on sclerocorneal buttons have described Type $2 \mathrm{BB}$ starting in the periphery, owing to the presence of clusters of fenestrations in the periphery of the impervious PDL. ${ }^{9,11}$ In this case series, Type 2 BB started in the center in 13 eyes and in the midperiphery in one eye. Peripheral commencement was not observed in any of the studied eyes. An explanation is that $\mathrm{KC}$ is associated with disruption of the collagen lamellae, and degradation of elastin in the PDL renders it pervious to air, ${ }^{14,15}$ contrary to sclerocorneal buttons, with normal stromal microarchitecture, where air should pass through the fenestrations in the periphery of the PDL to form a Type 2 BB. ${ }^{11}$ Detecting the type of BB after air injection is a crucial step for a successful DALK surgery, as Type 2 $\mathrm{BB}$ has special techniques of handling different than Type $1 \mathrm{BB}^{16}$ Therefore, surgeons should not rely on the peripheral commencement of Type $2 \mathrm{BB}$ if the clear margin of the bubble was not evident owing to the small size of the bubble or the presence of stromal whitening.

Although Type 2 BB formed in sclerocorneal buttons was described to be of large diameter (mean, $10.2 \mathrm{~mm}$ ) [10], smaller size was reported in this study (mean, $7.4 \mathrm{~mm}$ ). This is attributed to slow stromal air injection during DALK to avoid possible bursting and to allow cessation of injection as soon as Type 2 BB was noticed. It has been demonstrated that the bursting pressure of Type $2 \mathrm{BB}$ is much lower than that of type 1.9,12 Limiting the size of the formed Type 2 BB may also hypothetically lower the possibility of double anterior chamber in the postoperative period, through decreasing the peripheral extension of the bubble. ${ }^{16}$
The duration of formation of Type 2 BB has not previously been discussed in the literature. The rapid formation of Type $2 \mathrm{BB}$ reported in this study (mean, $0.3 \pm 0.12 \mathrm{~s}$ ), compared with that of Type $1 \mathrm{BB}$ (mean, $1.9 \pm 1.1 \mathrm{~s}$ ), could be attributed to the loose interfacial matrix attachment between the DM and PDL, compared with the relatively strong attachment between PDL and the posterior stroma. ${ }^{15}$

An interesting observation is the presence of a minute intrastromal bubble preceding most of Type $2 \mathrm{BB}$, which usually pass unnoticed owing to the small size and the quick progression to the more obvious Type $2 \mathrm{BB}$. This intrastromal bubble may be a small Type 1 BB; Therefore, it could be concluded that the number of mixed bubbles is usually underestimated. Intraoperatively, this small bubble may guide the surgeon to the plane between the PDL and stroma, thereby avoiding the hazards of DM baring. ${ }^{16}$

Limitations of this study include the small sample size, which is owing to the rarity of BB formation without stromal whitening, especially in Type $1 \mathrm{BB}$ which is usually preceded by varying degrees of stromal whitening, depending on the corneal thickness and the proximity of air injection cannula to the DM. However, previous studies describing the criteria and dynamics of Type 1 and 2 bubbles have had a sample size between 22 and 57 sclerocorneal buttons and between 3 and 9 eyes with formed Type 2 BB., ${ }^{9,112}$ Therefore, we believe a sample size of 14 keratoconic eyes with Type 2 BB, starting in the center and midperiphery may be considered enough to conclude that Type 2 BB in eyes with keratoconus can start anywhere, not necessary in the periphery. Moreover, the study was limited to keratoconic eyes owing to the preserved corneal clarity in keratoconus, allowing analysis of the air path during $\mathrm{BB}$ formation. It was not possible to include eyes with corneal scars or stromal dystrophies in this study, as the scars usually obscure the process of BB formation. Another limitation is that the study was restricted to eyes with minimal stromal whitening, even though other mechanisms may be present in eyes with marked whitening. Other tools such as intraoperative optical coherence tomography may assist in studying these eyes, which was not available in this study.

In conclusion, eyes with advanced $\mathrm{KC}$ were associated with central commencements of Type 1 and Type 2 BB during DALK using the BB technique. Unlike previously described in vitro, peripheral commencement of Type 2 BB was not observed in keratoconic eyes. This may prove the central affection of the PDL in advanced $\mathrm{KC}$, allowing air path to gain access to the plane between the DM and PDL. 


\section{Data Sharing Statement}

On request from Mohamed Bahgat Goweida. Any Data intended for sharing will be de-identified.

\section{Funding}

This research received no specific grants or funds.

\section{Disclosure}

The authors declare that they have no conflicts of interest.

\section{References}

1. Sugita J, Kondo J. Deep lamellar keratoplasty with complete removal of pathological stroma for vision improvement. $\mathrm{Br} J$ Ophthalmol. 1997;81(3):184-188. doi:10.1136/bjo.81.3.184

2. Cheng YY, Visser N, Schouten JS, et al. Endothelial cell loss and visual outcome of deep anterior lamellar keratoplasty versus penetrating keratoplasty: a randomized multicenter clinical trial. Ophthalmology. 2011;118(2):302-309. doi:10.1016/j.ophtha.20 10.06 .005

3. Han DCY, Mehta JS, Por YM, Htoon HM, Tan DTH. Comparison of outcomes of lamellar keratoplasty and penetrating keratoplasty in keratoconus. Am J Ophthalmol. 2009;148(5):744-51.e1. doi:10.10 16/j.ajo.2009.05.028

4. Anwar M, Teichmann KD. Big-bubble technique to bare Descemet's membrane in anterior lamellar keratoplasty. $J$ Cataract Refract Surg. 2002;28(3):398-403. doi:10.1016/S0886-3350(01)01181-6

5. Fogla R, Padmanabhan P. Results of deep lamellar keratoplasty using the big-bubble technique in patients with keratoconus. $\mathrm{Am}$ J Ophthalmol. 2006;141(2):254-259. doi:10.1016/j.ajo.2005.08.064

6. Fontana L, Parente G, Tassinari G. Clinical outcomes after deep anterior lamellar keratoplasty using the big-bubble technique in patients with keratoconus. Am J Ophthalmol. 2007;143(1):117-124. doi:10.1016/j.ajo.2006.09.025
7. Jafarinasab MR, Rahmati-Kamel M, Kanavi MR, Feizi S. Dissection plane in deep anterior lamellar keratoplasty using the big-bubble technique. Cornea. 2010;29(4):388-391. doi:10.1097/ ICO.0b013e3181ba7016

8. McKee HD, Irion LC, Carley FM, Jhanji V, Brahma AK. Residual corneal stroma in big-bubble deep anterior lamellar keratoplasty: a histological study in eye-bank corneas. Br J Ophthalmol. 2011;95 (10):1463-1465. doi:10.1136/bjophthalmol-2011-300360

9. Dua HS, Faraj LA, Said DG, Gray T, Lowe J. Human corneal anatomy redefined: a novel pre-Descemet's layer (Dua's layer). Ophthalmology. 2013;120(9):1778-1785. doi:10.1016/j.ophtha.20 13.01.018

10. Anwar M. Big-bubble technique. In: Luigi F, editor. Atlas of Lamellar Keratoplasty. San Giovani, Italy: Fabiano; 2007:125-136.

11. Dua HS, Faraj LA, Kenawy MB, et al. Dynamics of big bubble formation in deep anterior lamellar keratoplasty by the big bubble technique: in vitro studies. Acta Ophthalmol. 2018;96(1):69-76. doi:10.1111/aos. 13460

12. AlTaan SL, Mohammed I, Said DG, Dua HS. Air pressure changes in the creation and bursting of the type- 1 big bubble in deep anterior lamellar keratoplasty: an ex vivo study. Eye. 2018;32(1):146-151. doi:10.1038/eye.2017.121

13. Goweida MBB. Intraoperative review of different bubble types formed during pneumodissection (big-bubble) deep anterior lamellar keratoplasty. Cornea. 2015;34(6):621-624. doi:10.1097/ ICO.0000000000000407

14. White TL, Lewis PN, Young RD, et al. Elastic microfibril distribution in the cornea: differences between normal and keratoconic stroma. Exp Eye Res. 2017;159:40-48. doi:10.1016/j.exer.2017.03.002

15. Mohammed I, Ross AR, Britton JO, Said DG, Dua HS. Elastin Content and Distribution in Endothelial Keratoplasty Tissue Determines Direction of Scrolling. Am $J$ Ophthalmol. 2018;194:16-25. doi:10.1016/j.ajo.2018.07.001

16. Goweida MB, Ragab AM, Liu C. Management of Type 2 Bubble Formed During Big Bubble Deep Anterior Lamellar Keratoplasty. Cornea. 2019;38(2):189-193.
Clinical Ophthalmology

\section{Publish your work in this journal}

Clinical Ophthalmology is an international, peer-reviewed journal covering all subspecialties within ophthalmology. Key topics include: Optometry; Visual science; Pharmacology and drug therapy in eye diseases; Basic Sciences; Primary and Secondary eye care; Patient Safety and Quality of Care Improvements. This journal is indexed on PubMed
Central and CAS, and is the official journal of The Society of Clinical Ophthalmology (SCO). The manuscript management system is completely online and includes a very quick and fair peer-review system, which is all easy to use. Visit http://www.dovepress.com/ testimonials.php to read real quotes from published authors.

\section{Dovepress}

